\title{
Posthearing Developmental Refinement of Temporal Processing in Principal Neurons of the Medial Superior Olive
}

\author{
Luisa L. Scott, ${ }^{\star}$ Paul J. Mathews, ${ }^{\star}$ and Nace L. Golding \\ Section of Neurobiology and Institute for Neuroscience, University of Texas at Austin, Austin, Texas 78712-0248
}

\begin{abstract}
In mammals, principal neurons of the medial superior olive (MSO) exhibit biophysical specializations that enable them to detect soundlocalization cues with microsecond precision. In the present study, we used whole-cell patch recordings to examine the development of the intrinsic electrical properties of these neurons in brainstem slices from postnatal day 14 (P14) to P38 gerbils. In the week after hearing onset (P14-P21), we observed dramatic reductions in somatic EPSP duration, input resistance, and membrane time constant. Surprisingly, somatically recorded action potentials also dramatically declined in amplitude over a similar period (38 \pm 3 to $17 \pm 2 \mathrm{mV} ; \tau=$ $5.2 \mathrm{~d}$ ). Simultaneous somatic and dendritic patch recordings revealed that these action potentials were initiated in the axon, which primarily emerged from the soma. In older gerbils, the rapid speed of membrane voltage changes and the attenuation of action potential amplitudes were mediated extensively by low voltage-activated potassium channels containing the Kv1.1 subunit. In addition, whole-cell voltage-clamp recordings revealed that these potassium channels increase nearly fourfold from P14 to P23 and are thus a major component of developmental changes in excitability. Finally, the electrophysiological features of principal neurons of the medial nucleus of the trapezoid body did not change after P14, indicating that posthearing regulation of intrinsic membrane properties is not a general feature of all time-coding auditory neurons. We suggest that the striking electrical segregation of the axon from the soma and dendrites of MSO principal neurons minimizes spike-induced distortion of synaptic potentials and thus preserves the accuracy of binaural comparisons.
\end{abstract}

Key words: MSO; auditory brainstem; dendrotoxin; dendrite; MNTB; Kv1.1

\section{Introduction}

The medial superior olive (MSO) is one of several brainstem auditory nuclei that comprise the first stage for processing sound localization cues. MSO neurons compute the horizontal location of low-frequency sounds using the difference in the time required for a sound to propagate to each ear. These interaural time delays (ITDs) are submillisecond cues, the physiological range of which is dependent on the diameter of the animal's head. The principal neurons of the MSO are able to extract these brief ITDs from converging binaural inputs that include an excitatory component from the ipsilateral and contralateral ventral cochlear nucleus (VCN) (Cant and Casseday, 1986; Smith et al., 1993; Beckius et al., 1999) and an inhibitory component from both the medial nucleus (MNTB) and lateral nucleus (LNTB) of the trapezoid body (Helfert et al., 1989; Adams and Mugnaini, 1990; Cant and Hyson, 1992; Vater, 1995). Although the mechanism for extracting ITDs from these excitatory and inhibitory inputs has yet to be fully elucidated (for review, see Joris et al., 1988; Grothe, 2003; Palmer, 2004), ITD information is conveyed to higher auditory centers through a rate code (Goldberg and Brown, 1969; Yin and

\footnotetext{
Received March 15, 2005; revised July 16, 2005; accepted July 18, 2005.

We thank Sukant Khurana for helpful comments regarding this manuscript, as well as Bradley McKethan, Natalie Britt, and Erik Henserling for histological assistance.

*L.L.S. and P.J.M. contributed equally to this work.

Correspondence should be addressed to Nace L. Golding, Section of Neurobiology, C0920, University of Texas at Austin, Austin, TX 78712-0248. E-mail: golding@mail.utexas.edu.

D0I:10.1523/JNEUROSCI.1016-05.2005

Copyright $\odot 2005$ Society for Neuroscience $\quad$ 0270-6474/05/257887-09\$15.00/0
}

Chan, 1990; Spitzer and Semple, 1995; Brand et al., 2002). To detect these rapid time-varying cues, time-coding auditory neurons in the MSO and elsewhere in the brainstem exhibit biophysical specializations, such as low voltage-activated potassium currents $\left(I_{\mathrm{K}(\mathrm{LVA})}\right)$, which allow them to signal with high temporal fidelity (Svirskis et al., 2004) (for review, see Trussell, 1999).

Although the auditory canal opens at approximately postnatal day 12 (P12) in gerbils, rats, and mice, auditory function is not mature from the outset. In gerbils, the sound intensity required to produce responses by the cochlea and VCN neurons declines during the first week of hearing (Woolf and Ryan, 1984, 1985; Echteler et al., 1989). Additionally, the maximum firing rates of VCN and superior olivary neurons increase and tuning matures in these neurons as well as the cochlea (Harris and Dallos, 1984; Woolf and Ryan, 1985; Sanes and Rubel, 1988; Echteler et al., 1989). In humans and other mammals, the ability to localize sound sources on the horizontal plane also matures after hearing onset (Kelly et al., 1987; Ashmead et al., 1991).

Less attention has focused on the development of the intrinsic properties of MSO neurons. In fact, few intracellular recordings have been reported in the MSO beyond P20 (but see Smith, 1995). In the present study, we describe developmental changes in the properties of MSO principal neurons after hearing onset (P14-P38). During the first week of hearing, the membrane time constant decreases and EPSPs narrow. Action potentials, initiated in the axon, also decrease in amplitude. These developmental changes are mediated primarily by an increase in $I_{\mathrm{K}(\mathrm{LVA})}$. Although MNTB principal neurons also express $I_{\mathrm{K}(\mathrm{LVA})}$ (Banks and 
Smith, 1992; Brew and Forsythe, 1995; Dodson et al., 2002), their intrinsic properties do not change after hearing onset. Thus, the developmental refinement of electrical properties is not a general feature of all time-coding neurons.

\section{Materials and Methods}

Slice preparation. Mongolian gerbils (Meriones unguiculatus) were obtained from Charles River Laboratories (Wilmington, MA) or bred at the Animal Resource Center of the University of Texas at Austin. Gerbils (P14-P38) were anesthetized with halothane, and the brain was removed while submerged in oxygenated artificial CSF (ACSF) (in mM: $125 \mathrm{NaCl}$, $2.5 \mathrm{KCl}, 2 \mathrm{CaCl}_{2}, 25 \mathrm{NaHCO}_{3}, 1 \mathrm{MgSO}_{4}, 1.25 \mathrm{NaH}_{2} \mathrm{PO}_{4}$, and 25 glucose), $\mathrm{pH}$ 7.4, with $\mathrm{NaOH}$. Horizontal sections $(200 \mu \mathrm{m})$ were cut at $32^{\circ} \mathrm{C}$ using an oscillating tissue slicer (VT-1000S; Leica, Solms, Germany) and were then transferred to an incubating chamber containing oxygenated ACSF at $35^{\circ} \mathrm{C}$. After $30 \mathrm{~min}$, slices were held at room temperature until recording. Individual slices were transferred to a recording stage and bathed with oxygenated ACSF maintained at $35^{\circ} \mathrm{C}$ during recording.

Whole-cell recording. $\mathrm{MSO}$ and MNTB neurons were visualized using infrared differential interference contrast microscopy (Axioskop 2FS Plus; Zeiss, Oberkochen, Germany) in combination with a Newvicon tube camera (Dage-MTI, Michigan City, IN). Recordings were made using heat-polished borosilicate patch pipettes $(1.65 \mathrm{~mm}$ outer diameter; World Precision Instruments, Sarasota, FL) with open tip resistances of 2-4 M $\Omega$ (somatic electrodes) and 6-11 M $\Omega$ (dendritic electrodes). The internal solution in patch pipettes contained the following (in mM): 115 potassium gluconate, $20 \mathrm{KCl}, 10$ sodium phosphocreatine, 10 HEPES, 0.5 EGTA, 4 MgATP, $0.3 \mathrm{NaGTP}$, pH 7.3, with KOH. For current-clamp recordings, biocytin $(0.1 \%)$ was also included in the internal solution for subsequent morphological analyses of the recorded cells.

Somatic and dendritic recordings were made with Dagan (Minneapolis, MN) BVC-700A amplifiers in current-clamp mode. Activation of synaptic inputs to MSO and MNTB cells was achieved by delivering brief $(100 \mu \mathrm{s})$ electrical pulses to the slice through patch pipettes broken to tip diameters of $\sim 10 \mu \mathrm{m}$. Pulses were generated by a constant current stimulator (Digitimer, Welwyn Garden City, UK). Stimulation electrodes were placed both medial and lateral to the MSO. The identification of recorded cells was made on the basis of their location in the slice, cell body shape, and responses to depolarizing current steps. Additionally, many cells were successfully labeled with biocytin and their morphology examined (see below). To determine the effect of $I_{\mathrm{K}(\mathrm{LVA})}$ on the intrinsic properties of MSO neurons, 40-80 nм dendrotoxin-K (DTX-K; Alomone Labs, Jerusalem, Israel) was bath applied. To test the impact of the developmental increase in $I_{\mathrm{K}(\mathrm{LVA})}$ on temporal summation, two simulated EPSCs (biexponential waveform, $0.2 \mathrm{~ms}$ rise, and $0.2 \mathrm{~ms}$ decay, 3.5 $\mathrm{ms}$ duration) separated by $0-2.5 \mathrm{~ms}(0.1 \mathrm{~ms}$ increments $)$ were injected at the soma. Recordings were included if the series resistance was $<15 \mathrm{M} \Omega$ for somatic recordings and $<50 \mathrm{M} \Omega$ for dendritic recordings. Data were low-pass filtered at $5 \mathrm{kHz}$ and acquired at $50 \mathrm{kHz}$ using custom macros programmed in IgorPro Carbon (WaveMetrics, Lake Oswego, OR).

Somatic whole-cell voltage-clamp recordings were made with an Axopatch 200B amplifier (Molecular Devices, Union City, CA). Pipettes were wrapped with Parafilm (Pechiney Plastic Packaging, Chicago, IL) to reduce electrode capacitance. In addition to cell location and soma shape, MSO principal neurons were identified by their transient responses to suprathreshold depolarizing current steps in fast current-clamp mode. Slices were bathed in ACSF (above) with $10 \mathrm{~mm}$ tetraethylammonium chloride (TEA-C1), $1 \mu \mathrm{M}$ TTX, $200 \mu \mathrm{M} \mathrm{CoCl}_{2}, 1 \mu \mathrm{M}$ strychnine (Sigma, St. Louis, MO), $10 \mu \mathrm{M}$ CNQX, and $50 \mu \mathrm{M}$ ZD7288 (4-ethylphenylamino1,2-dimethyl-6-methylaminopyrimidinium chloride; Tocris Cookson, Ellisville, MO) to block high voltage-activated potassium, voltage-gated sodium and calcium channels, glycine and AMPA receptors, and $I_{\mathrm{h}}$, respectively. $\mathrm{NaCl}$ and dextrose were adjusted to 115 and $11 \mathrm{~mm}$, respectively, to maintain an osmotic difference of $20 \mathrm{mmol} / \mathrm{kg}$ between the external and internal solutions. DTX-K $(80 \mathrm{~nm})$ was later added to test the contribution of low voltage-activated potassium channels. The perfusion lines were cleaned with $0.1 \mathrm{M} \mathrm{NaOH}$ for 20 min between these experiments. The series resistance $(4-10 \mathrm{M} \Omega$ ) was compensated by at least $95 \%$, and the resting membrane potential was adjusted to account for a liquid junction potential of $+10 \mathrm{mV}$. The voltage offset was checked at the end of each recording to ensure a $<5 \mathrm{mV}$ offset. The data were low-pass filtered at $5 \mathrm{kHz}$ and acquired using custom macros for IgorPro at a sampling rate of $50 \mathrm{kHz}$.

Anatomy. Slices with biocytin-labeled cells were fixed in 4\% paraformaldehyde and subsequently processed using an ABC kit (nickelenhanced DAB reaction; Vector Laboratories, Burlingame, CA). MSO and MNTB principal neurons were verified by their location in the slice and by cell morphology. Cells were traced at $40 \times$ using an Axioskop FS2 with a camera lucida. Axon location with respect to the center of the soma was also measured at $40 \times$ using a Zeiss Axiocam CCD camera and Openlab 3.1.4 software (Improvision, Lexington, MA).

Analysis. All analysis was completed in IgorPro. The membrane time constant was obtained from a single exponential fit to the peak of a hyperpolarizing voltage response (3-5 $\mathrm{mV}$ from rest). Peak and steadystate $V-I$ plots were generated from voltage responses to $100 \mathrm{~ms}$ current steps measured at the peak and during the last $10 \mathrm{~ms}$ of the step pulse, respectively. The input resistance was obtained from the slope of the peak $V-I$ plot between 0 and $10 \mathrm{mV}$ below rest. Action potential amplitude was measured relative to the inflection point. The rise time of synaptically evoked EPSPs was measured between 10 and $90 \%$ of the peak amplitude, which was adjusted to $4-6 \mathrm{mV}$. The duration of these EPSPs was measured at half-maximal amplitude. In the voltage-clamp recordings, peak current responses were evoked from a $-80 \mathrm{mV}, 750 \mathrm{~ms}$ prepulse to voltages up to $0 \mathrm{mV}(1 \mathrm{~s})$. Leak current was extrapolated from peak responses to small depolarizing voltage steps from $-80 \mathrm{mV}$. Whole-cell capacitance was obtained from the amplifier after compensating for the series resistance, pipette capacitance, and whole-cell resistance and capacitance. Means are presented \pm SEM and compared using a two-way ANOVA or Student's $t$ test.

\section{Results}

To examine the maturation of intrinsic membrane properties and action potential signaling in the MSO, we made somatic whole-cell current-clamp recordings from 156 principal neurons in slices from P14-P38 gerbils. Neurons of all ages responded to suprathreshold depolarizing current steps with a single action potential accompanied by strong outward rectification, presumably reflecting the activation of voltage-gated potassium channels (Fig. 1A). Input resistances were extremely low in MSO principal neurons, which resulted in fast membrane time constants of $<2$ ms (Fig. 1 $A, B$ ). EPSPs exhibited fast kinetics, with rise times of $<330 \mu$ s and halfwidths of $<1.23$ ms. The rapid EPSP kinetics enabled MSO principal neurons to fire at high frequencies, up to hundreds of impulses per second, without temporal summation (Fig. 1C).

The duration of EPSPs was developmentally regulated in MSO principal neurons (Fig. 2). At P14-P16, soon after the opening of the auditory canal, EPSP width averaged $0.78 \pm 0.05$ $\mathrm{ms}(n=14)$, measured at half-maximal amplitude. Although the rate of rise for EPSPs remained relatively stable between P14 and P38 (Fig. 2A,B), in the week after hearing onset, the duration of EPSPs declined exponentially with a time constant of $4.3 \mathrm{~d}$ (Fig. $2 A, C)$. The input resistance and membrane time constant also declined over the same time window, reaching asymptotic values of $\sim 7 \mathrm{M} \Omega$ and $0.3 \mathrm{~ms}$ by $\sim 3$ weeks after birth (Fig. $2 D$ ). This developmental shortening of the time constant constrains the duration of the EPSP to more accurately reflect the time course of the underlying EPSC.

The developmental decline in EPSP duration, input resistance, and membrane time constant were significantly different when comparing means for P14-P16 and P19-P21 (EPSP duration, $p<0.01$; input resistance, $p<0.001$; membrane time constant, $p<0.001 ; n=7-18)$. There was no additional change in these parameters between P19-P21 and P28-P30. Also, the EPSP 

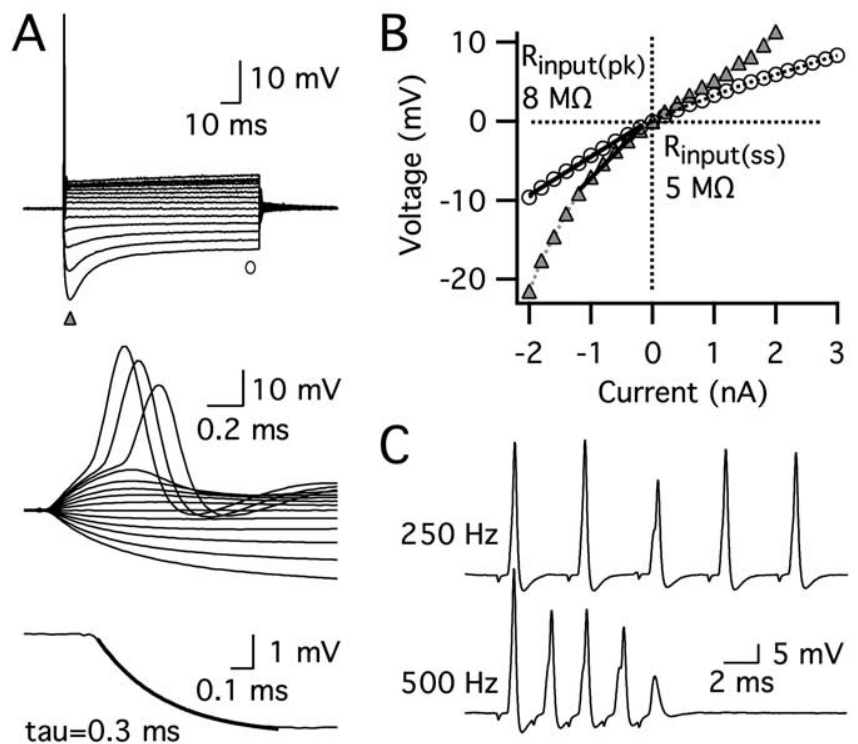

Figure 1. The intrinsic properties of an MSO principal neuron from a P21 gerbil. $\boldsymbol{A}$, Responses to a family of 100 ms current pulses ( -2 to $2.8 \mathrm{nA} ; 0.2 \mathrm{nA}$ steps). The resting membrane potential was $-58 \mathrm{mV}$. The universal response of MSO principal neurons to a suprathreshold depolarizing current step was a single action potential with fast repolarization (top and middle panels). Rapid voltage responses reflect a short membrane time constant, measured using a single exponential fit of a small hyperpolarizing voltage response (bottom panel). $\boldsymbol{B}$, Peak (triangles) and steady-state (circles) $V-I$ plots generated from the voltage responses shown in $A$ indicate that this neuron also had a low input resistance. Input resistance was obtained from the slope of the $V-I$ plot between 0 and $10 \mathrm{mV}$ below rest $\left(R_{\text {input(pk) }}=8 \mathrm{M} \Omega, R_{\text {input(ss) }}=5\right.$ $M \Omega$ ). C, Responses to synaptic stimulation in another cell (P19). MSO principal neurons followed high-frequency synaptic stimulation without temporal summation.

rate of rise did not change between these three age groups, $\mathrm{P} 14-$ P16, P19-P20, and P28-P30 ( $p>0.2 ; n=7-14)$.

The shape and amplitude of action potentials in MSO principal neurons also exhibited striking changes during the first week of hearing (Fig. 3). At P14, action potentials were typically large and exceeded $0 \mathrm{mV}$, similar to action potentials in most brain areas. In slices from older animals, action potentials appeared progressively smaller in amplitude, with slower rates of rise, and larger current thresholds for initiation (Fig. $3 A-D$ ). In electrophysiologically mature neurons, action potentials were small near threshold (between 5 and $15 \mathrm{mV}$ ) but were graded with increasing stimulus amplitude (Fig. $1 \mathrm{~A}$, middle traces). These small action potentials could double in amplitude in response to strong synaptic excitation (data not shown).

When compared across age groups, action potential amplitudes and maximum rates of rise decreased by $>50 \%$ between P14-P16 and P19-P21 [amplitude, $p<0.001$; time derivative of voltage $(\mathrm{dV} / \mathrm{dt}), p<0.001 ; n=16-21]$, whereas the current required for initiation nearly doubled over the same period $(p<$ $0.001 ; n=16-20)$. Unlike the input resistance and membrane time constant, action potential parameters continued to change moderately between P19-P21 and P28-P30. During this period, the action potential amplitudes and maximum rates of rise decreased again by almost 50\% (amplitude, $p<0.01$; dV/dt, $p<$ $0.05 ; n=7-16)$ and the current required for initiation nearly doubled ( $p<0.05 ; n=6-16$ ). In contrast with the increase in current required for action potential initiation, the voltage threshold declined by $\sim 7 \mathrm{mV}$ after hearing onset with the majority of this decline occurring between P19-P21 and P28-P30 (P19-P21 vs P28-P30, $p<0.01$; P14-P16, -31.4 $\pm 1.16 \mathrm{mV}$;
$\mathrm{P} 19-\mathrm{P} 21,-33.3 \pm 4.83 \mathrm{mV}$; P28-P30, $-38.2 \pm 1.33 \mathrm{mV} ; n=$ 6-21).

Developmental changes in action potential amplitude could be caused by a shift in the balance of sodium and potassium currents after hearing onset. Alternatively, the site of action potential initiation could shift farther from the soma. To distinguish between these two possibilities, we examined action potential initiation through morphological and electrophysiological analyses. First, we determined where the axon emerged from the cell by analyzing the morphology and axon location of 129 biocytinlabeled neurons (Fig. 4A). In most cells, the axon emerged directly from the soma $(73 \%)$, whereas the remaining axons typically emerged from the proximal dendrite, $<30 \mu \mathrm{m}$ from the soma (8\% medial, 19\% lateral) (Fig. 4B). This pattern was observed at all ages included in our study.

To address whether action potential initiation occurs in the axons of MSO principal neurons, we made dual current-clamp recordings at the soma and proximal dendrite, $<50 \mu \mathrm{m}$ from the soma in P16-P19 gerbils. Action potentials were initiated with either somatic current injection or synaptic stimulation. When the axon emerged from the soma $(n=5$ of 6$)$, action potentials evoked by somatic current injection were always detected first at the soma and subsequently in the dendrite in an attenuated form (Fig. 4C, top left). This was also true for synaptically evoked action potentials, although in one case, the peak timing was ambiguous because of the broader shape in combination with a short recording distance (Fig. 4C, bottom left). However, when the axon emerged from the lateral dendrite within $5 \mu \mathrm{m}$ of the dendritic recording site ( $50 \mu \mathrm{m}$ from the soma; $n=1$ of 6 ), the action potential was detected first in the dendrite (Fig. $4 C$, right column). The range of action potential amplitudes in these dual recordings (17-56 $\mathrm{mV}$ at the soma) suggests that both small and large action potentials initiate near or in the axon. Together, the anatomical and physiological results are consistent with an axonal site of action potential initiation, independent of electrophysiological maturity. Thus, developmental changes in action potential amplitude and shape are more likely attributable to changes in the balance of voltage-dependent conductances.

To test the role of $I_{\mathrm{K}(\mathrm{LVA})}$ in action potential generation, we examined the effects of the Kv1.1-containing channel blocker DTX-K on MSO principal neurons exhibiting mature electrophysiological properties (P20-P21 gerbils). Although the transient firing pattern of five neurons was not altered in the presence of 40 or $80 \mathrm{~nm}$ DTX-K (Fig. $5 \mathrm{~A}$ ), the toxin induced dramatic changes in other response properties. The membrane time constant and input resistance increased approximately threefold, and action potential amplitude increased from $15 \pm 3.6$ to $37 \pm 4.9$ $\mathrm{mV}$ (Fig. 5B-F). DTX-K reduced the threshold current for action potential initiation from $2.7 \pm 0.19$ to $0.9 \pm 0.09 \mathrm{nA}$ (Fig. $5 D, F)$. These results show that Kv1.1-containing channels contribute strongly to the low input resistance and fast membrane time constant, enabling these channels to play a key role in the rapid signaling of MSO principal neurons.

Because $I_{\mathrm{K}(\mathrm{LVA})}$ shapes the membrane time constant and action potential amplitude, this current is a likely candidate for the change in electrical properties during the first week of hearing. We tested the idea that $I_{\mathrm{K}(\mathrm{LVA})}$ is developmentally regulated by pharmacologically isolating this current during whole-cell voltage-clamp recordings of $49 \mathrm{MSO}$ principal neurons (see Materials and Methods). Depolarizing voltage steps elicited fastactivating and slow-inactivating outward currents (Fig. 6A, top). Peak $I_{\mathrm{K}(\mathrm{LVA})}$ increased almost fourfold between P14 and P23 (Fig. $6 C)$, with no significant change in voltage sensitivity of activation 


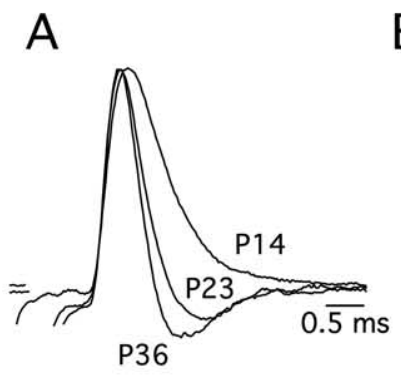

B

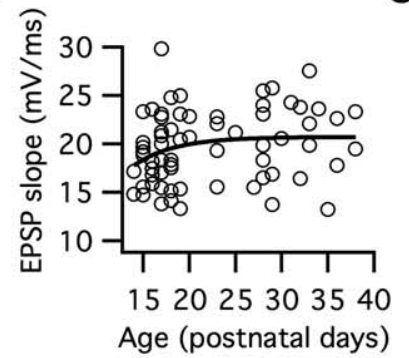

C

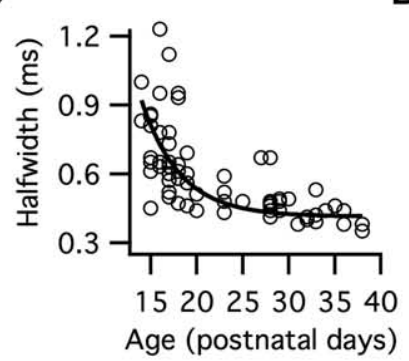

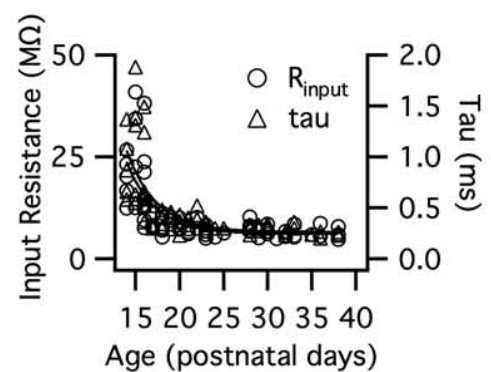

Figure 2. The physiological properties of MSO principal neurons undergo developmental changes after hearing onset. A, Synaptically evoked EPSPs (4-6mV) with normalized amplitudes at P14, P23, and P36 show that EPSP shape is developmentally regulated. Strychnine $(1 \mu \mathrm{m})$ blocked glycinergic inhibition during all cases of synaptic stimulation. $\boldsymbol{B}$, The rate of rise for EPSPs remained relatively stable after hearing onset $(n=67)$. $\boldsymbol{C}$, In the same neurons as in $\boldsymbol{B}$, the duration of EPSPs, measured at half-maximal amplitude, declined nearly one-third between P14 and P21 with a single exponential time constant of $4.3 \mathrm{~d}$. D. The input resistance (left axis; circles) and membrane time constant (right axis; triangles) of 68 principal neurons declined threefold over the same time window, both with time constants of $3.3 \mathrm{~d}$ (single exponential fit). The fits for input resistance (black) and membrane time constant (gray) essentially overlap.

A
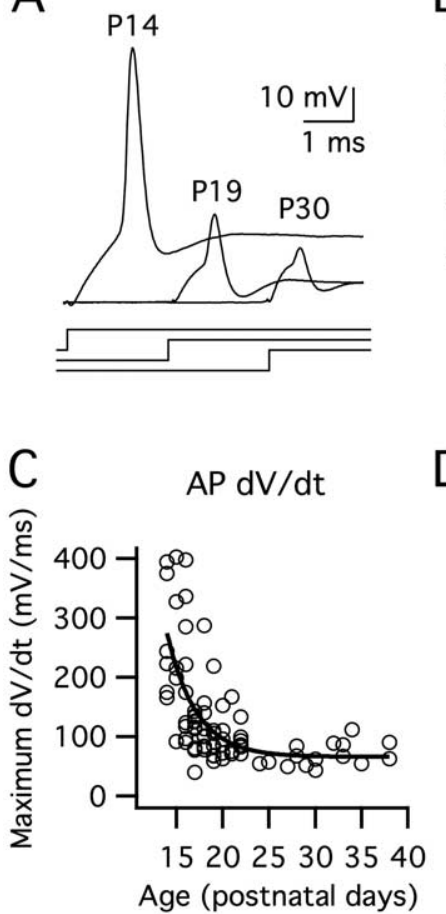

B

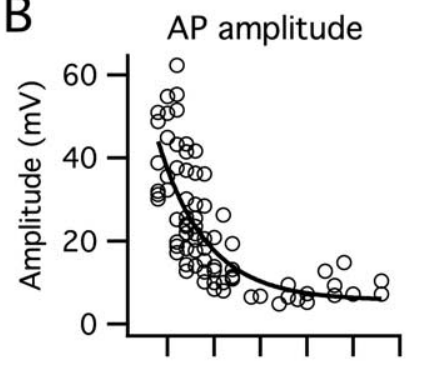

152025303540

Age (postnatal days)
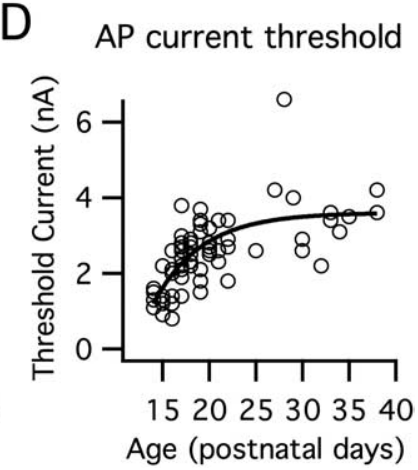

Figure 3. The shape and amplitude of action potentials in MSO principal neurons progressively change after hearing onset. Action potentials were evoked in 81 cells via suprathreshold $100 \mathrm{~ms}$ square current steps. $\boldsymbol{A}$, At P14, action potentials were large, exceeding $0 \mathrm{mV}$. Over the next few days, action potential amplitudes and rates of rise decreased markedly. Current steps are shown below the action potential responses. $\boldsymbol{B}$, Action potential amplitudes, measured with respect to the inflection point, declined with a single exponential time constant of $5.2 \mathrm{~d}$. $\boldsymbol{C}$, Maximum rates of rise decreased with a time constant of $3.1 \mathrm{~d}$. $D$, The current required for action potential initiation increased with a time constant of $5.2 \mathrm{~d}$ (single exponential fit). AP, Action potential.

(data not shown). Changes in cell size or membrane area do not account for this developmental increase in $I_{\mathrm{K}(\mathrm{LVA})}$ because wholecell capacitance did not significantly change $(<1 \%)$ between P14 and P23 (Fig. 6D).

At both $\mathrm{P} 14$ and $\mathrm{P} 21, I_{\mathrm{K}(\mathrm{LVA})}$ was reduced significantly by 80 nM DTX-K (Fig. 6A, $B$ ), showing $I_{\mathrm{K}(\mathrm{LVA})}$ is mediated predominantly by Kv1.1-containing channels in the week after hearing onset. Interestingly, the average percentage block by DTX-K increased from $76 \%$ at $\mathrm{P} 14$ to $91 \%$ at $\mathrm{P} 21$ (steps from -80 to -45 $\mathrm{mV}$ ) (Fig. $6 E$ ), suggesting that the proportion of potassium chan-

nels containing the Kv1.1 subunit increases during development. The time constant for the activation of this DTX-sensitive current decreased with age $(\mathrm{P} 14, \tau=1.47 \pm 0.17 \mathrm{~ms} ; \mathrm{P} 21, \tau=0.95 \pm 0.10$ $\mathrm{ms}, p<0.05$; steps from -80 to $-45 \mathrm{mV}$ ). This faster activation kinetics at P21 than P14 could contribute to the developmental change in action potential amplitude by decreasing the ratio of inward-to-outward current during action potential generation. However, the action potentials themselves were initiated earlier at P21 versus P14 (0.70 ms and $1.03 \mathrm{~ms}$, respectively), which would, in part, offset these effects. A precise quantification of the influence of channel kinetics on action potential amplitude cannot be made based on these experiments. Together, the developmental changes in the density and possibly also the kinetics of $I_{\mathrm{K}(\mathrm{LVA})}$ are critical factors underlying the developmental maturation of intrinsic properties and action potential signaling in early posthearing development.

To gain insight into how the developmental upregulation of Kv1.1-mediated currents shapes the temporal resolution of excitatory synaptic integration, two artificial EPSCs separated by a variable delay $(0-2.5 \mathrm{~ms}$, in $0.1 \mathrm{~ms}$ steps) were combined and injected somatically into cells from P14 and P21 animals. The amplitude of a single EPSC was adjusted to evoke an $\sim 3 \mathrm{mV}$ EPSP, and the peak voltage of paired responses were normalized to this value. In normal saline, the window for temporal integration (defined as the time taken for averaged EPSPs to repolarize to within $10 \%$ of baseline) was twice as narrow at P21 versus P14 $(0.87 \pm 0.04 \mathrm{~ms}$ vs $1.64 \pm 0.23 \mathrm{~ms} ; p<0.05)$ (Fig. $7 B, C)$. This difference reflects the shorter membrane time constant in older animals. To isolate the contribution of $I_{\mathrm{K}(\mathrm{LVA})}$ upregulation to the developmental change in EPSP summation, we used DTX-K on cells from P21 animals to block $I_{\mathrm{K}(\mathrm{LVA})}$ to a similar level found in cells from P14 animals (in separate voltage-clamp experiments, $31 \mathrm{~nm}$ DTX-K blocked $73.5 \pm 1.8 \%$ of $I_{\mathrm{K}(\mathrm{LVA})}$ ) (Fig. $7 A$ ). In the presence of $31 \mathrm{~nm}$ DTX-K, the window for temporal summation increased to a value comparable with that observed in cells from P14 animals (P21 in DTX-K, $1.72 \pm 0.09 \mathrm{~ms}$; P14, $1.64 \pm 0.23$ $\mathrm{ms}$ ) (Fig. $7 B, C$ ). Together, these findings show that the agerelated increase in $I_{\mathrm{K}(\mathrm{LVA})}$ significantly sharpens the window for temporal integration.

Kv1.1-mediated currents are found in nearly all brainstem auditory neurons concerned with temporal coding and have been studied most extensively in MNTB principal neurons (Brew and Forsythe, 1995; Grigg et al., 2000; Dodson et al., 2002; Brew et al., 2003; Barnes-Davies et al., 2004; Leao et al., 2004). To address whether the properties of MNTB neurons change after hearing 


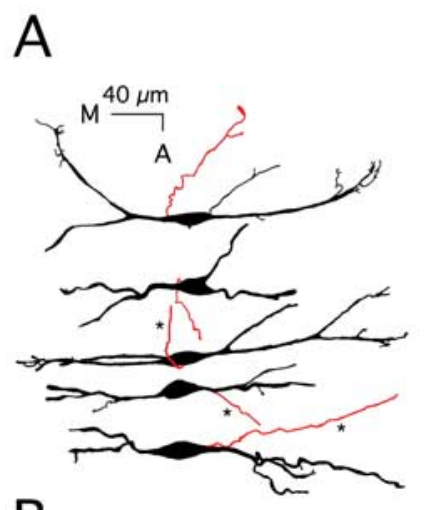

B
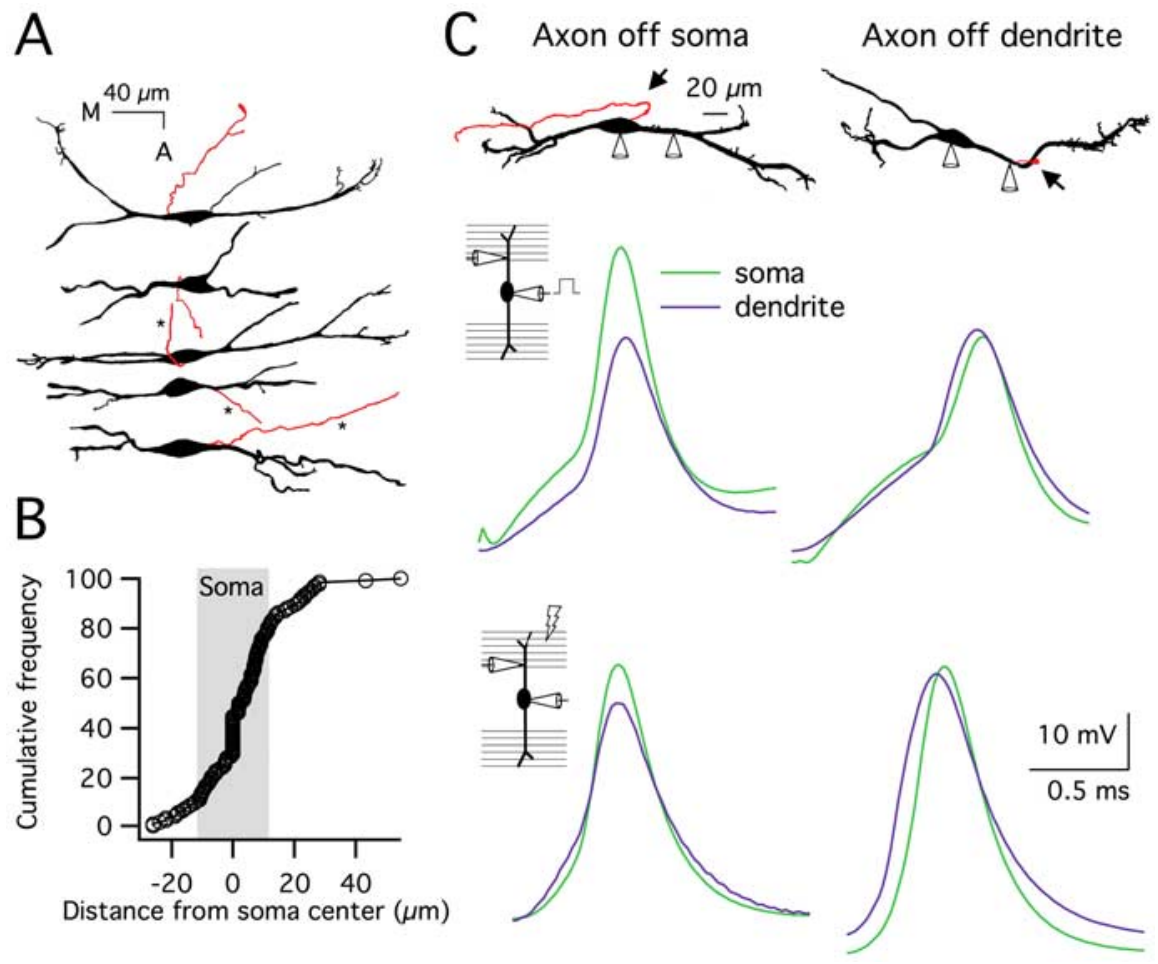

Figure 4. Action potentials are initiated in the axon of MSO principal neurons, and axon location does not change as a function of age. $\boldsymbol{A}$, Camera lucida reconstructions of biocytin-filled MSO neurons show axon location (in red). Three axons were truncated for the figure $\left({ }^{*}\right)$. B, A cumulative frequency plot of 129 cells shows that most axons emerged directly from the soma $(73 \%)$, as indicated by the points within the shaded region. In the remaining cells, $19 \%$ of the axons emerged proximally from the lateral dendrite (to the right of the shaded region), and $8 \%$ emerged from the medial dendrite within $8 \mu \mathrm{m}$ from the soma (to the left of the shaded region). C, Dual somatic and proximal dendritic current-clamp recordings in two neurons, one with a somatically located axon (left) and one with an axon extending from the lateral dendrite $\sim 50 \mu \mathrm{m}$ from the soma (right). Top panel, Camera lucida reconstructions show the location of the axons (in red and indicated by arrows). For the neuron with a dendritically located axon, the dendritic recording site and the axon were $<5 \mu \mathrm{m}$ apart. In both neurons, the medial dendrite is on the left. Middle and bottom panels, Somatic (green traces) and dendritic (lateral dendrite; purple traces) responses to suprathreshold somatic current injection (middle panels) or lateral synaptic stimulation (bottom panels). For the neuron with an axon extending from the soma, action potentials were detected first at the soma and then $35 \mu \mathrm{m}$ out on the dendrite in an attenuated form (lag time, $20 \mu \mathrm{s}$ for current injection, $0 \mu \mathrm{s}$ for synaptic stimulation). However, in the neuron with the dendritically located axon, action potentials were first detected at the dendritic recording site, $50 \mu \mathrm{m}$ from the soma, and then at the soma (lag time, $20 \mu \mathrm{s}$ for current injection, $40 \mu$ s for synaptic stimulation).

onset, we made whole-cell current-clamp recordings from 20 principal neurons between the ages of P14 and P37. Although the morphology of MNTB principal neurons is distinct from MSO neurons (Fig. $8 \mathrm{~A}$ ), they exhibit many of the same general electrophysiological features, including a phasic firing pattern and strong outward rectification (Fig. 8B) (Banks and Smith, 1992; Brew and Forsythe, 1995). Our recordings from MNTB principal neurons in gerbils generally were similar to previous studies but revealed significant electrophysiological differences from their MSO counterparts present in the same slices. MNTB principal cells fired large action potentials that were greater in amplitude and required far more modest currents (average across all ages, $0.47 \mathrm{nA}$ ) for initiation relative to principal neurons of the MSO (Fig. $8 D, E$ ). The input resistance and membrane time constant were also up to 12 -fold higher in MNTB than in MSO principal neurons (e.g., Fig. 8C). Although the intrinsic properties of MSO principal neurons changed dramatically in the first 3 weeks of hearing, they did not change significantly in MNTB principal cells even up to P37.

When we compared across age groups, action potential amplitude and threshold were constant in MNTB principal neurons between P14-P16, P19-P21, and P28-P30 ( $p>0.05)$ (Fig.
$8 D, E)$. Overall, MNTB principal neurons had large action potentials and required modest currents for action potential initiation relative to principal neurons of the MSO (amplitude, $p<0.001, n=6-21$; threshold, $p<0.001, n=6-20)$. The peak input resistance and membrane time constant of MNTB principal cells also remained stable after hearing onset (P14$\mathrm{P} 16,131 \pm 33.2 \mathrm{M} \Omega, 3.9 \pm 0.75 \mathrm{~ms} ; \mathrm{P} 19-$ $\mathrm{P} 20,115 \pm 20.0 \mathrm{M} \Omega, 4.3 \pm 0.60 \mathrm{~ms} ; \mathrm{P} 28-$ $\mathrm{P} 29,86 \pm 13 \mathrm{M} \Omega, 3.6 \pm 0.72 \mathrm{~ms} ; p>0.4$ $n=6-7)$. Furthermore, the values of these membrane characteristics were larger than those of the MSO neurons (input resistance, $p<0.001, n=6-18$; membrane time constant, $p<0.001, n=6-18)$. (Banks and Smith, 1992; Brew and Forsythe, 1995)

\section{Discussion}

Principal neurons of the medial superior olive detect interaural time differences introduced by sounds in the environment and encode these cues in their action potential firing rate. The resolution of this computation relies in part on the speed and brevity of excitatory synaptic transmission. In the present study, we describe the temporal refinement of action potential signaling and subthreshold electrical properties of MSO neurons in gerbils. The week after hearing onset, action potential amplitudes decrease considerably, and a decline in EPSP duration mirrors a reduction in membrane time constant. These changes are driven primarily by an increase in $I_{\mathrm{K}(\mathrm{LVA})}$, which in turn is mediated by channels containing the Kv1.1 subunit. Finally, these developmental changes in the MSO are not universal to all brainstem auditory neurons concerned with temporal coding, because MNTB principal neurons exhibit stable intrinsic electrical properties and action potential signaling after the onset of hearing.

\section{Properties and developmental regulation of $I_{\mathrm{K}(\mathrm{LVA})}$ in MSO principal neurons}

We show that, in principal neurons of the MSO, a developmentally regulated expression of low-voltage potassium channels plays an important role in the signaling of microsecond differences in synaptic timing. From 2 to 3 weeks of age, a decrease in average EPSP halfwidth from 0.78 to $0.55 \mathrm{~ms}$ is temporally correlated with a nearly fourfold increase in $I_{\mathrm{K}(\mathrm{LVA})}$. One possible mechanism underlying this change in EPSP duration is an acceleration in AMPA receptor kinetics, which has been described in studies of other auditory brainstem synapses (Bellingham et al., 1998; Lawrence and Trussell, 2000; Taschenberger and von Gersdorff, 2000; Joshi et al., 2004; MacLeod and Carr, 2005). However, this issue has not yet been addressed in the MSO. The developmental increase in $I_{\mathrm{K}(\mathrm{LVA})}$ decreases EPSP duration in two ways. First, tonic $I_{\mathrm{K}(\mathrm{LVA})}$ activation near rest decreases input resistance, thereby increasing the intrinsic speed of membrane volt- 

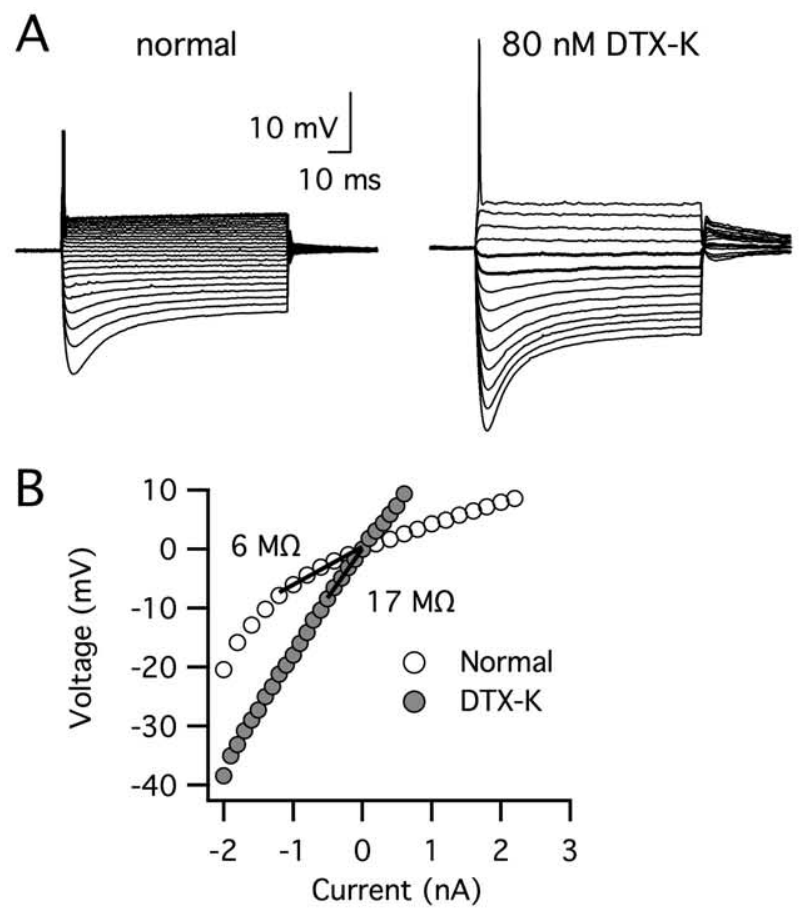
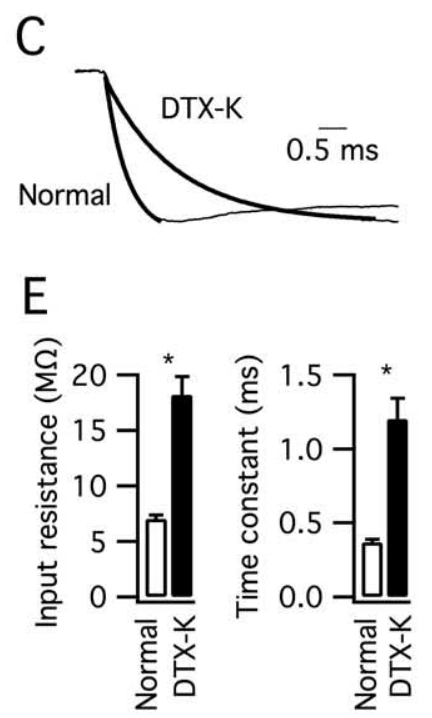

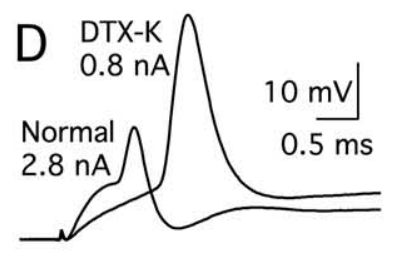

$\mathrm{F}$

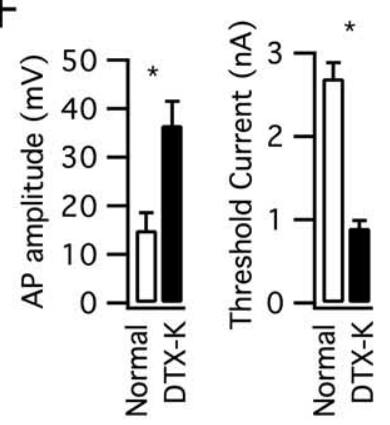

Figure 5. The application of DTX-K altered the electrophysiological properties of MSO principal neurons exhibiting mature features (P20-P21). $A$, The response of a typical MSO neuron to current pulses ( -2 to 0.8 or $2.8 \mathrm{nA}, 0.2 \mathrm{nA}$ steps) before and after the application of $80 \mathrm{~nm}$ DTX-K shows that a transient firing pattern persisted in the presence of the toxin. $\boldsymbol{B}$, In contrast, the slope of the peak $V-I$ plot below rest reveals that the input resistance of this cell increased $>2.5$-fold. C, Normalized inward voltage responses show that the neuron's membrane time constant also increased threefold from 0.4 to $1.3 \mathrm{~ms}$ after toxin application. $\boldsymbol{D}$, Action potentials in this neuron increased in amplitude and the threshold current for action potential initiation decreased in response to DTX-K. E, $\boldsymbol{F}$, Average membrane time constant, peak input resistance, threshold current for action potential initiation and action potential amplitude before (open bars) and after (filled bars) DTX-K application $(n=5)$. All parameters were significantly different in DTX-K ( $\left.{ }^{*} p<0.001\right)$. AP, Action potential. Error bars represent SEM.

age changes by threefold. Second, $I_{\mathrm{K}(\mathrm{LVA})}$ activation by synaptic depolarization actively repolarizes the EPSP and truncates the duration of the response (our unpublished observations). Regardless of the mechanism, the developmental decrease in EPSP duration narrows the window for temporal summation of EPSPs by a factor of two (Fig. 7), enhancing the cycle-to-cycle indepen-

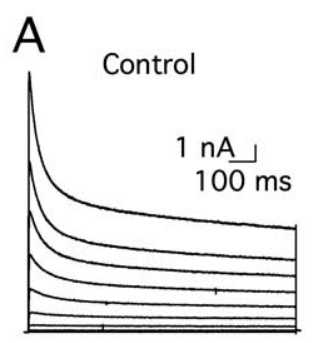

80nM DTX-K
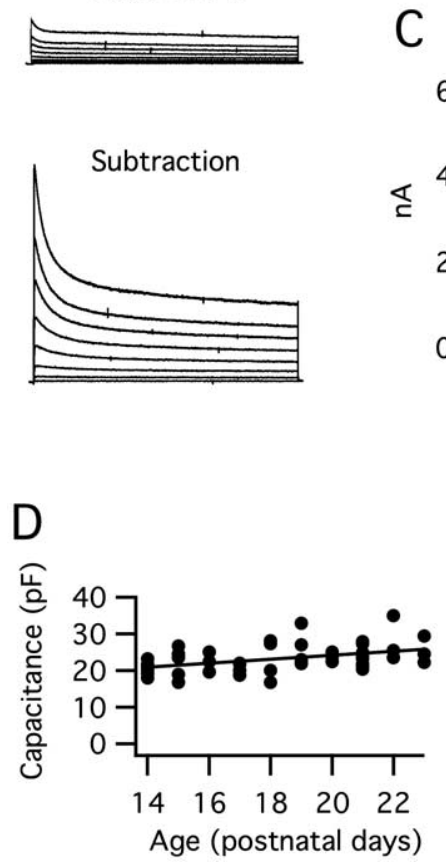

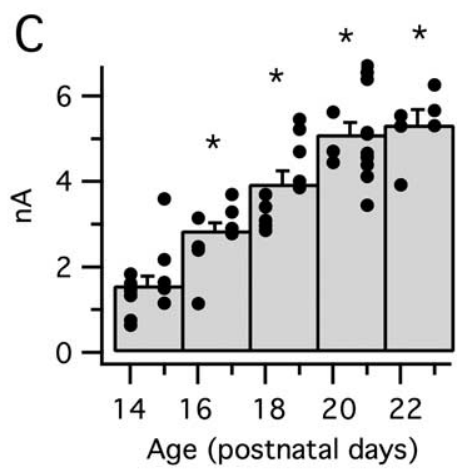

E

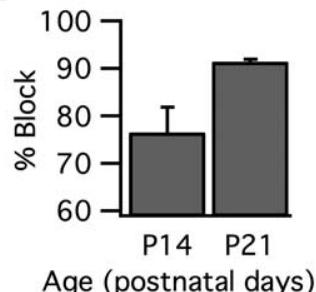

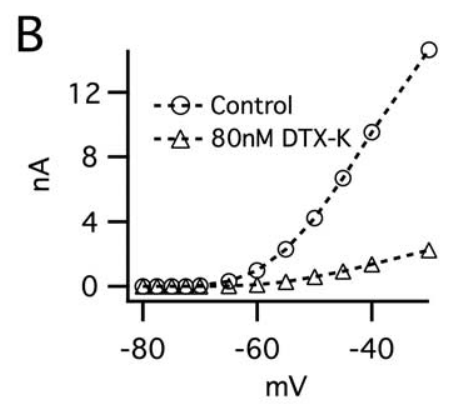

Figure 6. Low voltage-activated potassium current $\left(I_{\mathrm{K}(\mathrm{LVA})}\right)$ increases after hearing onset in MSO principal neurons. $I_{\mathrm{K}(\mathrm{LVA})}$ was isolated pharmacologically (see Materials and Methods) and evoked by a family of $1 \mathrm{~s}$ depolarizations $(-70$ to $-30 \mathrm{mV} ; 5 \mathrm{mV}$ steps; from a prepulse potential of $-80 \mathrm{mV}$ ). $\boldsymbol{A}$, In a typical neuron from P21, $I_{\mathrm{K}(\mathrm{LVA})}$ (top) was essentially blocked by the addition of $80 \mathrm{~nm}$ DTX-K (middle). DTX-K-sensitive currents are shown in the bottom panel. Traces are not leak-subtracted. B, Peak $/-V$ curves for leak-subtracted $I_{\mathrm{K}(\mathrm{LVA})}$ before (circles) and after (triangles) the addition of $80 \mathrm{~nm}$ DTX-K (the same neuron as $\boldsymbol{A}$ ). For this neuron, $I_{\mathrm{K}(\mathrm{LVA})}$ was blocked by $80-90 \%$ across the range of voltages shown. C, Peak $I_{\text {K(LVA) }}$ (leak-subtracted) as a function of age. This current increases $390 \%$ between $P 14$ and P23. The histogram displays peak current responses for voltage steps to $-45 \mathrm{mV}$, grouped in $2 \mathrm{~d}$ bins. The superimposed filled circles represent currents from individual cells. The asterisks indicate significant difference from the P14-P15 group; $p<0.001$. D, Whole-cell capacitance did not change significantly between P14 and P23, indicating that changes in cell size/membrane area do not account for the developmental increase in $I_{K(L V A)}$. $E$, DTX-K $(80 \mathrm{~nm})$ blocks the majority of $I_{K(L V A)}$ in response to steps to $-45 \mathrm{mV}$. The percentage block increases from 76 to $91 \%$ between P14 and P21 ( $p=$ $0.06 ; n=4-6)$. Error bars represent SEM.

dence of binaural integration in the face of high-frequency synaptic activity. Increased $I_{\mathrm{K}(\mathrm{LVA})}$ also imparts a threefold higher current threshold for action potential initiation, increasing the requirement for synchronicity of binaural excitation.

A similar increase in $I_{\mathrm{K}(\mathrm{LVA})}$ has been reported in neurons of the nucleus laminaris (Kuba et al., 2002), the avian homolog of the MSO, suggesting that developmental upregulation of $I_{\mathrm{K} \text { (LVA) }}$ is a common feature of binaural coincidence detectors in birds and mammals. However, unlike in chick nucleus laminaris (Smith and Rubel, 1979; Kuba et al., 2005), we did not find electrophysiological or morphological changes along the putative tonotopic axis in the MSO. The reason that electrical maturity is 
A
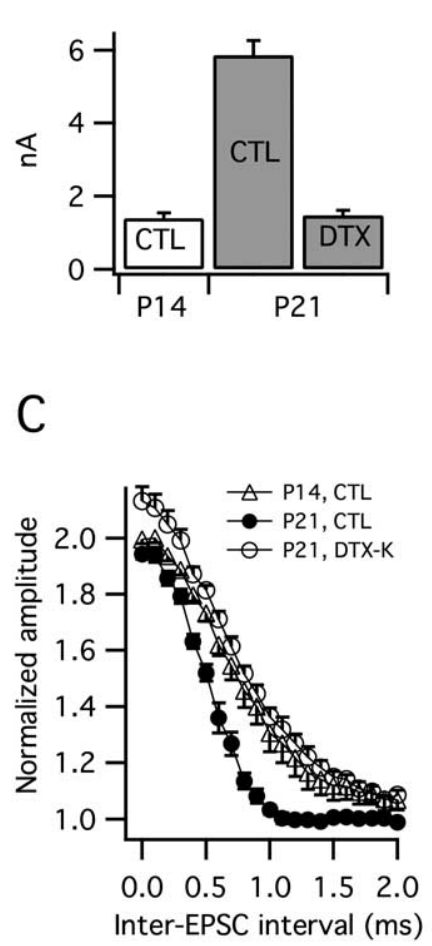

B

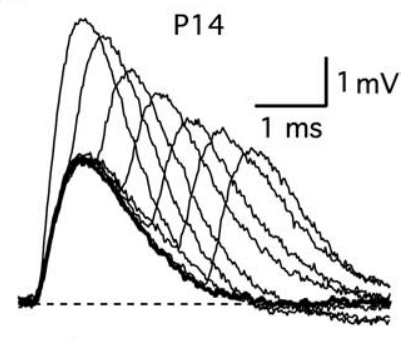

P21
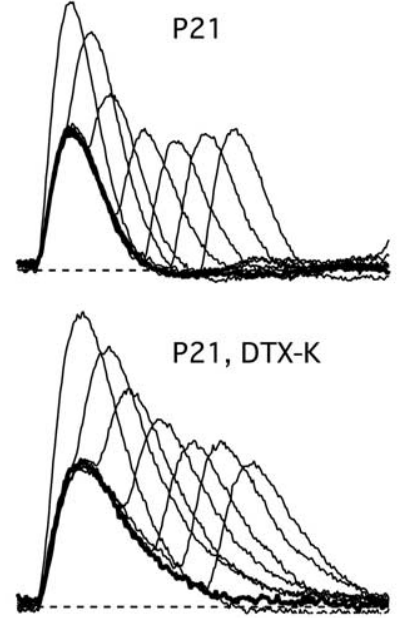

Figure 7. The developmental increase in $I_{\mathrm{K}(L \mathrm{VA})}$ narrows the window for temporal summation. $A, I_{\mathrm{K}(L \mathrm{~V}) \mathrm{C}}$ measured in whole-cell voltage-clamp recordings increases approximately fourfold between P14 and P21 (amplitude shown for steps to $-45 \mathrm{mV}$ from a $-80 \mathrm{mV}$ prepulse). Bath application of $31 \mathrm{~nm}$ DTX-K partially blocked $I_{K(L V A)}$ by $73.5 \pm 1.8 \%$ rendering the amplitude of $I_{K(L V A)}$ at $P 21$ similar to the amplitude at $P 14$. B. Current-clamp responses to single (thick lines) and paired simulated EPSC injections (pairs separated by $0-2 \mathrm{~ms}, 0.4 \mathrm{~ms}$ steps, thin lines) at P14 and P21. Partial block of DTX-K-sensitive current ( $31 \mathrm{~nm}$ DTX-K) widened responses at P21 such that they were similar to those at P14. Single EPSPs were adjusted to $\sim 3 \mathrm{mV}$. C, Peak voltage responses to paired EPSCS. Summed responses were normalized to the peak amplitudes of single EPSPs. The window for temporal summation was larger at P14 than at P21 $(p<0.01)$. The reduction of DTX-K sensitive current with $31 \mathrm{~nm}$ DTX-K broadened the window for temporal integration at P21 $(p<0.001)$. The window of temporal integration was defined as the time taken for averaged EPSP amplitudes to fall within $10 \%$ of baseline. CTL, Control.

delayed until a week after hearing onset in MSO principal neurons is not clear. One possibility for this delay is that channel expression may not be preprogrammed, but rather can be influenced by sound-induced afferent activity. Consistent with this idea, in cultured hippocampal pyramidal neurons, Kv1.1- and Kv1.2-containing potassium channels are upregulated during growth in culture through a process that is sensitive to synaptic activity (Grosse et al., 2000). Few other studies have examined the postnatal expression of Kv1-containing channels in detail. However, the robust upregulation in channel expression observed in the MSO is not a general feature of neural development, because Kv1-containing channels in chick vestibular neurons appear to undergo a decrease in expression after hatching (Gamkrelidze et al., 2000; Popratiloff et al., 2003). Also, in the present study, there was no significant change in Kv1.1-related electrical properties in MNTB principal neurons between P14 and P38.

In MSO principal neurons, the majority of $I_{\mathrm{K}(\mathrm{LVA})}$ was blocked by $80 \mathrm{~nm}$ DTX-K, indicating that Kv1.1-containing channels are a primary constituent of this outward current. The voltage sensitivity, kinetics, and dendrotoxin sensitivity of $I_{\mathrm{K}(\mathrm{LVA})}$ in MSO principal cells in many respects resembles that of other brainstem auditory neurons concerned with maintaining the precise timing
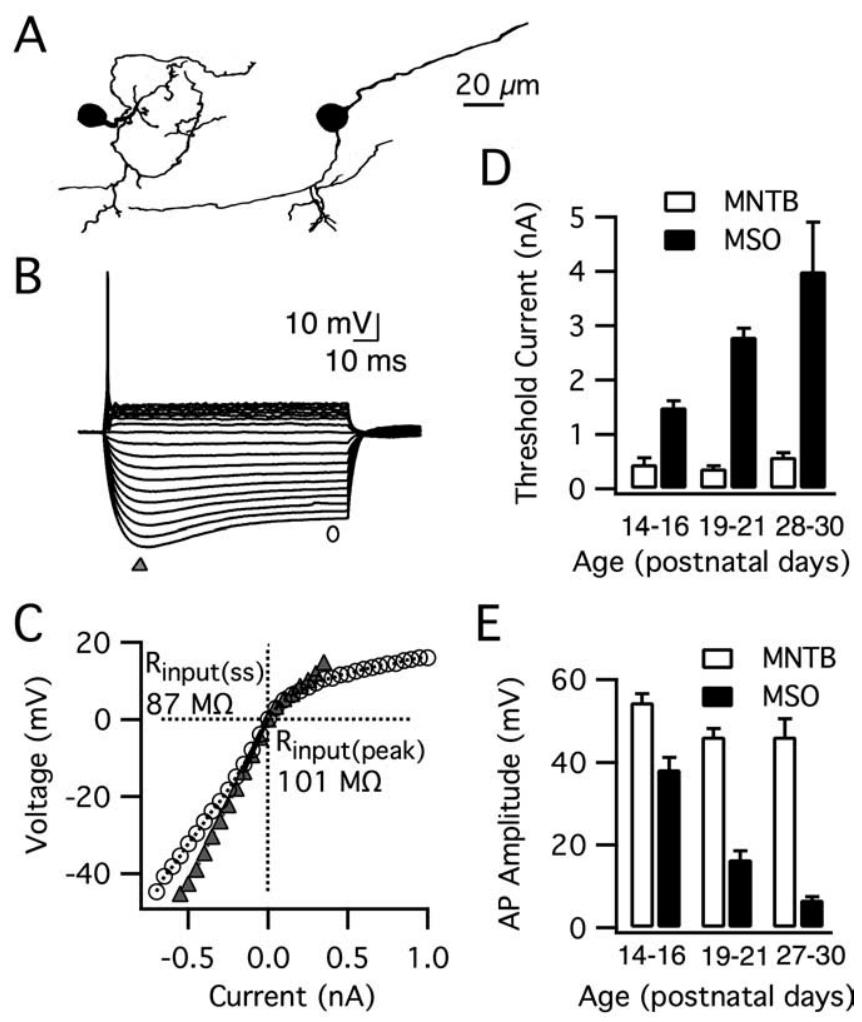

Figure 8. Principal neurons of the MNTB do not undergo changes in their intrinsic properties after hearing onset. $\boldsymbol{A}$, Camera lucida tracings of two biocytin-labeled MNTB principal neurons show their characteristic ovoid somata and small-caliber dendrites. $\boldsymbol{B}$, Responses to step pulses ( -0.55 to $0.35 \mathrm{nA}, 0.05 \mathrm{nA}$ steps) for a typical MNTB principal cell (P15; resting membrane potential, $-68 \mathrm{mV}$ ). MNTB principal neurons exhibit a transient firing pattern with strong outward rectification, similar to MSO principal neurons (see Fig. $1 A$ ). C, The peak (triangle) and steady-state (circle) $V$ - I plot for this MNTB principal neuron demonstrates that the input resistance near rest of MNTB neurons is larger than MSO neurons. The input resistance was obtained from the slope of the $V-/$ plot between 0 and $10 \mathrm{mV}$ below rest $\left(R_{\text {input(pk) }}=101 \mathrm{M} \Omega, R_{\text {input(ss) }}\right.$ $=87 \mathrm{M} \Omega$ ). $\boldsymbol{D}, \boldsymbol{E}$, MNTB principal cells fired large action potentials $2-4$ weeks after birth with no significant change in the threshold current for initiation ( $p>0.05 ; n=6-7$ ) or amplitude ( $p>0.1 ; n=6-7)$. In contrast, the action potential signaling properties of MSO principal neurons changed dramatically (threshold current, $p<0.001$; amplitude, $p<0.001 ; n=$ $7-21, n=7-20)$. Overall, MNTB principal neurons required far more modest currents for action potential initiation and had larger action potentials relative to principal neurons of the MSO (threshold, $p<0.001, n=6-20$; amplitude, $p<0.001, n=6-21$ ).

of excitatory inputs, such as bushy and octopus cells of the VCN, and principal cells of the MNTB (Manis and Marx, 1991; Rathouz and Trussell, 1998; Bal and Oertel, 2001; Dodson et al., 2002; Rothman and Manis, 2003). In situ hybridization and immunocytochemical analyses also reveal that the Kv1.1 subunit is prominently expressed in these brainstem auditory nuclei (Grigg et al., 2000; Dodson et al., 2002; Lu et al., 2004). In the present study, we observed an increase in the amount of $I_{\mathrm{K}(\mathrm{LVA})}$ blocked by DTX-K between P14 and P21 (76 to 91\% block), possibly reflecting an increase in the proportion of channels containing Kv1.1.

\section{Action potential initiation in MSO principal neurons}

Morphological analyses revealed that axons emerge primarily from the soma, and secondarily from the proximal dendrites of gerbil MSO principal neurons [see also the study by Smith (1995) in guinea pigs]. Our data also indicate that the site of action potential initiation is closely correlated with axon location in these neurons. During simultaneous somatic and dendritic recordings, action potentials were always detected first at the re- 
cording site closest to the axon. These results are in agreement with evidence from multiple types of central neurons indicating that action potentials initiate in the axon (Colbert and Pan, 2002) and subsequently propagate down the axon as well as back into the soma and dendrites (for review, see Häusser et al., 2000). However, unlike other CNS neurons, action potentials in electrophysiologically mature MSO principal neurons are unusually small at the soma $(<15 \mathrm{mV})$. These action potentials increased 2.5 -fold in amplitude in the presence of DTX-K, showing that Kv1.1-mediated currents strongly shape action potential amplitude in MSO neurons. Our findings raise the possibility that low voltage-activated potassium channels in the axon itself attenuate conventional, large-amplitude action potentials as they backpropagate from the axon to the soma.

MSO principal neurons fire a single, well timed action potential in response to step depolarizations. Similar to our findings, Grothe and Sanes (1993) found that action potential amplitudes did not exceed 20-25 mV in MSO neurons from P17-P25 gerbils (measured from the inflection point). In contrast, Smith (1995) found large action potentials $\sim 50 \mathrm{mV}$ in amplitude in 3- to 5 -week-old guinea pigs. This latter discrepancy may reflect species differences in either the rate of development or mature firing properties. Previous studies of the MSO have highlighted the importance of voltage-gated potassium currents, particularly those active at low voltages, in shaping action potential signaling. For example, reducing $I_{\mathrm{K}(\mathrm{LVA})}$ in MSO principal neurons from P12-P14 gerbils with blockers of Kv1 subunits, DTX-K and dendrotoxin-I, lowered the current threshold for action potential initiation and caused repetitive firing (Svirskis et al., 2002, 2004). Similar effects were found in older animals with a less-specific blocker, $1 \mathrm{~mm}$ 4-aminopyridine (Smith, 1995). However, in agreement with Svirskis et al. (2002), we found that DTX-K application to MSO principal neurons from gerbils older than P14 lowered the current threshold for action potential initiation without altering firing patterns. Thus, $I_{\mathrm{K}(\mathrm{LVA})}$ is not the only mechanism shaping the transient firing pattern in mature MSO principal neurons.

DTX-sensitive potassium currents in hippocampal, neocortical, and cerebellar Purkinje neurons impact the threshold and timing of both sodium- and calcium-dependent spikes; however, unlike MSO neurons, these currents have little effect on spike amplitude and shape. Variations in the role of DTX-sensitive currents presumably reflect differences in the subunit composition of native Kv1 potassium channels and/or differences in relative channel density (Storm, 1988; Golding et al., 1999; Bekkers and Delaney, 2001; McKay et al., 2005).

\section{Two classes of time-coding auditory neurons?}

In contrast to the robust changes in intrinsic electrical properties of MSO neurons during the first week of hearing, the electrical properties of principal neurons of the MNTB were stable throughout the entire range of animal ages we used (P14-P37), and were also substantially different in magnitude. In older MNTB neurons (P28-P30), action potential amplitudes were sevenfold larger, and input resistance and membrane time constants were 12-fold larger than in MSO neurons, suggesting there are differences in the relative density and/or composition of conductances between these neuron types. Based on these observations, we suggest that principal neurons of the MSO and MNTB comprise two related but functionally distinct classes of timecoding auditory brainstem neurons.

In MSO principal neurons, $I_{\mathrm{K}(\mathrm{LVA})}$ electrically insulates the soma and dendrites, in which synaptic integration takes place, from the voltage and temporal distortions of axonal action potentials. This functional compartmentalization may be shared by other auditory brainstem neurons integrating precisely timed inputs in dendrites. For example, octopus cells of the VCN detect the synchrony of auditory nerve fiber inputs in their large-caliber dendrites (Oertel et al., 2000). In these cells, the membrane time constant and somatic action potential amplitude undergo a substantial decrease during the first week of hearing in mice (R. Bal and D. Oertel, personal communication). It will be intriguing to see whether this electrophysiological motif is observed in other auditory neurons concerned with submillisecond dendritic integration.

\section{References}

Adams JC, Mugnaini E (1990) Immunocytochemical evidence for inhibitory and disinhibitory circuits in the superior olive. Hear Res 49:281-298.

Ashmead DH, Davis DL, Whalen T, Odom RD (1991) Sound localization and sensitivity to interaural time differences in human infants. Child Dev 62:1211-1226.

Bal R, Oertel D (2001) Potassium currents in octopus cells of the mammalian cochlear nucleus. J Neurophysiol 86:2299-2311.

Banks MI, Smith PH (1992) Intracellular recordings from neurobiotinlabeled cells in brain slices of the rat medial nucleus of the trapezoid body. J Neurosci 12:2819-2837.

Barnes-Davies M, Barker MC, Osmani F, Forsythe ID (2004) Kv1 currents mediate a gradient of principal neuron excitability across the tonotopic axis in the rat lateral superior olive. Eur J Neurosci 19:325-333.

Beckius GE, Batra R, Oliver DL (1999) Axons from anteroventral cochlear nucleus that terminate in medial superior olive of cat: observations related to delay lines. J Neurosci 19:3146-3161.

Bekkers JM, Delaney AJ (2001) Modulation of excitability by $\alpha$-dendrotoxin-sensitive potassium channels in neocortical pyramidal neurons. J Neurosci 21:6553-6560.

Bellingham MC, Lim R, Walmsley B (1998) Developmental changes in EPSC quantal size and quantal content at a central glutamatergic synapse in rat. J Physiol (Lond) 511:861-869.

Brand A, Behrend O, Marquardt T, McAlpine D, Grothe B (2002) Precise inhibition is essential for microsecond interaural time difference coding. Nature 417:543-547.

Brew HM, Forsythe ID (1995) Two voltage-dependent $\mathrm{K}^{+}$conductances with complementary functions in postsynaptic integration at a central auditory synapse. J Neurosci 15:8011-8022.

Brew HM, Hallows JL, Tempel BL (2003) Hyperexcitability and reduced low threshold potassium currents in auditory neurons of mice lacking the channel subunit Kv1.1. J Physiol (Lond) 548:1-20.

Cant NB, Casseday JH (1986) Projections from the anteroventral cochlear nucleus to the lateral and medial superior olivary nuclei. J Comp Neurol 247:457-476.

Cant NB, Hyson RL (1992) Projections from the lateral nucleus of the trapezoid body to the medial superior olivary nucleus in the gerbil. Hear Res 58:26-34.

Colbert CM, Pan E (2002) Ion channel properties underlying axonal action potential initiation in pyramidal neurons. Nat Neurosci 5:533-538.

Dodson PD, Barker MC, Forsythe ID (2002) Two heteromeric Kv1 potassium channels differentially regulate action potential firing. J Neurosci 22:6953-6961.

Echteler SM, Arjmand E, Dallos P (1989) Developmental alterations in the frequency map of the mammalian cochlea. Nature 341:147-149.

Gamkrelidze G, Giaume C, Peusner KD (2000) Firing properties and dendrotoxin-sensitive sustained potassium current in vestibular nuclei neurons of the hatchling chick. Exp Brain Res 134:398-401.

Goldberg JM, Brown P (1969) Response of binaural neurons of dog superior olivary complex to dichotic tonal stimuli: some physiological mechanisms of sound localization. J Neurophysiol 32:613-636.

Golding NL, Jung HY, Mickus T, Spruston N (1999) Dendritic calcium spike initiation and repolarization are controlled by distinct potassium channel subtypes in CA1 pyramidal neurons. J Neurosci 19:8789-8798.

Grigg JJ, Brew HM, Tempel BL (2000) Differential expression of voltagegated potassium channel genes in auditory nuclei of the mouse brainstem. Hear Res 140:77-90.

Grosse G, Draguhn A, Hohne L, Tapp R, Veh RW, Ahnert-Hilger G (2000) 
Expression of Kvl potassium channels in mouse hippocampal primary cultures: development and activity-dependent regulation. J Neurosci 20:1869-1882.

Grothe B (2003) New roles for synaptic inhibition in sound localization. Nat Rev Neurosci 4:540-550.

Grothe B, Sanes DH (1993) Bilateral inhibition by glycinergic afferents in the medial superior olive. J Neurophysiol 69:1192-1196.

Harris DM, Dallos P (1984) Ontogenetic changes in frequency mapping of a mammalian ear. Science 225:741-743.

Häusser M, Spruston N, Stuart GJ (2000) Diversity and dynamics of dendritic signaling. Science 290:739-744.

Helfert RH, Bonneau JM, Wenthold RJ, Altschuler RA (1989) GABA and glycine immunoreactivity in the guinea pig superior olivary complex. Brain Res 501:269-286.

Joris PX, Smith PH, Yin TC (1988) Coincidence detection in the auditory system: 50 years after Jeffress. Neuron 21:1235-1238.

Joshi I, Shokralla S, Titis P, Wang L-Y (2004) The role of AMPA receptor gating in the development of high-fidelity neurotransmission at the Calyx of Held synapse. J Neurosci 24:183-196.

Kelly JB, Judge PW, Fraser IH (1987) Development of the auditory orientation response in the albino rat (Rattus norvegicus). J Comp Psychol 101:60-66.

Kuba H, Koyano K, Ohmori H (2002) Development of membrane conductance improves coincidence detection in the nucleus laminaris of the chicken. J Physiol (Lond) 540:529-542.

Kuba H, Yamada R, Fukui I, Ohmori H (2005) Tonotopic specialization of auditory coincidence detection in nucleus laminaris of the chick. J Neurosci 25:1924-1934.

Lawrence JJ, Trussell LO (2000) Long-term specification of AMPA receptor properties after synapse formation. J Neurosci 20:4864-4870.

Leao RN, Berntson A, Forsythe ID, Walmsley B (2004) Reduced low-voltage activated $\mathrm{K}+$ conductances and enhanced central excitability in a congenitally deaf (dn/dn) mouse. J Physiol (Lond) 559:25-33.

Lu Y, Monsivais P, Tempel BL, Rubel EW (2004) Activity-dependent regulation of the potassium channel subunits Kv1.1 and Kv3.1. JComp Neurol 470:93-106.

MacLeod KM, Carr CE (2005) Synaptic physiology in the cochlear nucleus angularis of the chick. J Neurophysiol 93:2520-2529.

Manis PB, Marx SO (1991) Outward currents in isolated ventral cochlear nucleus neurons. J Neurosci 11:2865-2880.

McKay BE, Molineux ML, Mehaffey WH, Turner RW (2005) Kv1 K ${ }^{+}$channels control Purkinje cell output to facilitate postsynaptic rebound discharge in deep cerebellar neurons. J Neurosci 25:1481-1492.

Oertel D, Bal R, Gardner SM, Smith PH, Joris PX (2000) Detection of synchrony in the activity of auditory nerve fibers by octopus cells of the mammalian cochlear nucleus. Proc Natl Acad Sci USA 97:11773-11779.

Palmer AR (2004) Reassessing mechanisms of low-frequency sound localisation. Curr Opin Neurobiol 14:457-460.
Popratiloff A, Giaume C, Peusner KD (2003) Developmental change in expression and subcellular localization of two shaker-related potassium channel proteins $(\mathrm{Kv} 1.1$ and $\mathrm{Kv1.2})$ in the chick tangential vestibular nucleus. J Comp Neurol 461:466-482.

Rathouz M, Trussell L (1998) Characterization of outward currents in neurons of the avian nucleus magnocellularis. J Neurophysiol 80:2824-2835.

Rothman JS, Manis PB (2003) Differential expression of three distinct potassium currents in the ventral cochlear nucleus. J Neurophysiol 89:3070-3082.

Sanes DH, Rubel EW (1988) The ontogeny of inhibition and excitation in the gerbil lateral superior olive. J Neurosci 8:682-700.

Smith DJ, Rubel EW (1979) Organization and development of brain stem auditory nuclei of the chicken: dendritic gradients in nucleus laminaris. J Comp Neurol 186:213-239.

Smith PH (1995) Structural and functional differences distinguish principal from nonprincipal cells in the guinea pig MSO slice. J Neurophysiol 73:1653-1667.

Smith PH, Joris PX, Yin TC (1993) Projections of physiologically characterized spherical bushy cell axons from the cochlear nucleus of the cat: evidence for delay lines to the medial superior olive. J Comp Neurol 331:245-260.

Spitzer MW, Semple MN (1995) Neurons sensitive to interaural phase disparity in gerbil superior olive: diverse monaural and temporal response properties. J Neurophysiol 73:1668-1690.

Storm JF (1988) Temporal integration by a slowly inactivating $\mathrm{K}^{+}$current in hippocampal neurons. Nature 336:379-381.

Svirskis G, Kotak V, Sanes DH, Rinzel J (2002) Enhancement of signal-tonoise ratio and phase locking for small inputs by a low-threshold outward current in auditory neurons. J Neurosci 22:11019-11025.

Svirskis G, Kotak V, Sanes DH, Rinzel J (2004) Sodium along with lowthreshold potassium currents enhance coincidence detection of subthreshold noisy signals in MSO neurons. J Neurophysiol 91:2465-2473.

Taschenberger H, von Gersdorff H (2000) Fine-tuning an auditory synapse for speed and fidelity: developmental changes in presynaptic waveform, EPSC kinetics, and synaptic plasticity. J Neurosci 20:9162-9173.

Trussell LO (1999) Synaptic mechanisms for coding timing in auditory neurons. Annu Rev Physiol 61:477-496.

Vater M (1995) Ultrastructural and immunocytochemical observations on the superior olivary complex of the mustached bat. J Comp Neurol 358:155-180.

Woolf NK, Ryan AF (1984) The development of auditory function in the cochlea of the mongolian gerbil. Hear Res 13:277-283.

Woolf NK, Ryan AF (1985) Ontogeny of neural discharge patterns in the ventral cochlear nucleus of the mongolian gerbil. Dev Brain Res 17:131-147.

Yin TC, Chan JCK (1990) Interaural time sensitivity in medial superior olive of cat. J Neurophysiol 64:465-488. 